CLINICAL STUDY

\title{
Restricted thyroglobulin antibody epitope specificities in subjects with type 1 diabetes mellitus
}

\author{
O E Okosieme, C N Wijeyaratne ${ }^{1}$, J H Lazarus ${ }^{2}$ and L D K E Premawardhana ${ }^{2,3}$ \\ Department of Endocrinology and Diabetes, Prince Charles Hospital, Cwm Taff NHS Trust, Merthyr Tydfil, Mid Glamorgan CF47 9DT, UK, ${ }^{1}$ Department of \\ Obstetrics and Gynaecology, Faculty of Medicine, Kynsey Road, Colombo 7, Sri Lanka, ${ }^{2}$ School of Medicine, Centre for Endocrine and Diabetes Sciences, \\ Cardiff University, Cardiff CF14 4XN, South Wales, UK and ${ }^{3}$ Department of Endocrinology and Diabetes, Caerphilly District Miner's Hospital, \\ St Martin's Road, Caerphilly CF83 2WW, UK
}

(Correspondence should be addressed to O E Okosieme; Email: onyebuchi.okosieme@wales.nhs.uk)

\begin{abstract}
Objectives: Following iodisation in Sri Lanka we observed a high prevalence of thyroglobulin antibodies (TgAbs) in type 1 diabetic (T1DM) patients. The clinical significance of these TgAbs is uncertain. We sought to obtain a detailed epitope analysis of TgAbs in T1DM patients recruited from diabetes clinics and to compare these with $\mathrm{TgAb}$ epitope specificities in patients with autoimmune thyroid disease (AITD) and healthy individuals in that country.

Design and methods: We used a panel of $10 \mathrm{Tg}-\mathrm{MAbs}$ in competitive ELISA reactions in a prospective study of subjects recruited from Colombo, to determine the epitopes recognised by TgAb-positive patients with T1DM $(n=58,34 \mathrm{~F}: 24 \mathrm{M}$, median age 16 years $)$, AITD patients $(n=42,33 \mathrm{~F}: 9 \mathrm{M}$, median age 37 years) and healthy subjects $(n=50,39 \mathrm{~F}: 11 \mathrm{M}$, median age 27 years). The outcomes were a comparison of reactivity with six $\mathrm{Tg}$ clusters (I-VI) in these subjects, and the relation of epitope specificity patterns with free thyroxine and TSH.

Results: Patients with T1DM and AITD but not healthy control subjects preferentially recognised the immunodominant clusters, I, III and IV. Patients with these narrow epitope specificities had higher median TSH levels (1.60 vs 1.06; $P=0.01$ ), and were more frequently positive for antibodies to thyroid peroxidase than those with broad specificities ( 52.3 vs $7.1 \% ; P=0.004$ ).

Conclusions: The TgAb epitope specificities in euthyroid Sri Lankans with T1DM are similar to AITD patients. TgAb epitope studies may potentially identify T1DM patients at risk of thyroid dysfunction.

European Journal of Endocrinology $161489-493$
\end{abstract}

\section{Introduction}

Type 1 diabetes (T1DM) and autoimmune thyroid disease (AITD) are classic examples of organ-specific autoimmunity. Both conditions frequently coexist and up to one-third of patients with T1DM develop thyroid dysfunction during long-term follow-up (1). AITD is characterised by the presence of circulating antibodies to thyroid peroxidase (TPOAb) and thyroglobulin $(\mathrm{Tg} A b)(2)$. These antibodies occur more frequently in patients with T1DM than in healthy individuals (3). However, thyroid antibodies are also present in about $10 \%$ of healthy euthyroid individuals (4) and have been reported in $30 \%$ of healthy persons in newly iodised populations $(5,6)$. In the wake of iodine prophylaxis in Sri Lanka, we observed a high prevalence of TgAbs in various population subgroups (6). These TgAbs were not associated with thyroid dysfunction and probably represented a non-pathological antibody response to iodination (6). Recently, we also reported a high frequency of $\mathrm{TgAbs}$ in Sri Lankan patients with T1DM (7). The clinical significance of TgAbs in these diabetic patients is thus unclear and a point of interest is whether they indicate thyroiditis or whether they simply represent a non-pathological effect of iodination.

Studies on $\mathrm{TgAb}$ epitope reactivity have provided a potential means of distinguishing the $\mathrm{TgAbs}$ seen in health from those seen in disease $(8,9) . \mathrm{Tg}$, a large molecular weight (660-kDa) glycoprotein, is the major colloid protein and serves as a prohormone and storage protein for the thyroid hormones, thyroxine $\left(\mathrm{T}_{4}\right)$ and triiodothyronine (10). Detailed panels of murine Tg-MAbs have delineated several B-cell epitopes on $\mathrm{Tg}$ that are differentially recognised by sera from healthy individuals and AITD patients (11). These immunodominant $\mathrm{Tg}$ epitopes are located in the less conserved non-hormonogenic portions of the $\mathrm{Tg}$ molecule and are preferentially recognised by sera from the majority of AITD patients. On the other hand, healthy euthyroid individuals exhibit a broad or less-restricted $\mathrm{Tg}$ epitope reactivity pattern $(8,9,12)$.

The TgAb epitope specificities in patients with T1DM are unknown, and to the best of our knowledge have not been previously tested in any population group. 
Knowledge of these specificities will shed more light on the significance of thyroid antibodies in T1DM and could potentially identify the subsets of diabetic patients at risk of thyroid dysfunction. Our objective in the present study was thus to obtain a detailed epitope analysis of TgAbs in Sri Lankan T1DM patients using a well-characterised panel of Tg-MAbs. Furthermore, we compared these $\mathrm{TgAb}$ patterns with the typical patterns seen in health and in thyroiditis by evaluating $\mathrm{TgAb}$ specificities in healthy euthyroid individuals and in patients with established AITD.

\section{Methods}

\section{Subjects}

We tested serum for TgAbs in 217 patients with T1DM attending clinics in Colombo, Sri Lanka, and selected all $58 \mathrm{TgAb}$-positive patients for $\mathrm{Tg}$ epitope analysis (34F:24M, median age 16, age range 5-35 years, median duration of diabetes 9.38 months, range 0.4-57.08 months). Details of the prevalence of other disease-associated antibodies in the group have been described elsewhere (7). T1DM was diagnosed according to WHO criteria (13). The AITD control group comprised $42 \mathrm{TgAb}$-positive Sri Lankan patients with Hashimoto's thyroiditis (HT) attending a thyroid clinic in Colombo (33F:9M; median age 37 years, age range 16-41 years). HT was diagnosed based on the presence of goitre, TgAbs and/or TPOAbs, and in some cases, thyroid-histological and/or ultrasound features of autoimmune thyroiditis. The healthy control group consisted of $50 \mathrm{TgAb}$-positive healthy blood donors (belonging to the ethnic Sinhala group) without thyroid disease or diabetes (39F:11M; median age 27 years, age range 18-39 years). Ethical permission for the project was obtained from the local Ethics Committee and informed consent was obtained from the subjects or their parents as required.

\section{Thyroid function tests}

We measured free $\mathrm{T}_{4}\left(\mathrm{FT}_{4}\right.$; normal 9.8-23 pmol/l) with a competitive labelled antibody assay and TSH (normal 0.35-5.2 mU/l) by a two-site immunochemiluminometric assay (Bayer Plc Diagnostics Division). These were analysed on an automated immunoassay analyser, the ACS-180 Plus (Chiron Diagnostics Ltd, Halstead, Essex, UK). The interassay coefficient of variation for $\mathrm{FT}_{4}$ was $4 \%$ (at a mean of $13.6 \mathrm{pmol} / \mathrm{l}$ ) and the variation for TSH was $7.56 \%$ (at a mean of $4.89 \mathrm{mU} / \mathrm{l}$ ).

\section{Autoantibody estimations}

We measured TgAb (normal $<98 \mathrm{kIU} / \mathrm{l}$ ) and TPOAb (normal $<19.4 \mathrm{kIU} / \mathrm{l}$ ) by an ELISA technique standardised against National Institute for Biological Standards and Control reference standards (14). Intra-assay variations were $4.1 \%$ for TPOAb (at a mean of $150 \mathrm{kIU} / \mathrm{l}$ ) and $4.4 \%$ for $\mathrm{TgAb}$ (at a mean of $1420 \mathrm{kIU} / \mathrm{l}$ ). The interassay variations were $7.2 \%$ for TPOAb (at a mean of $138 \mathrm{kIU} / \mathrm{l}$ ) and $6.7 \%$ for $\mathrm{TgAb}$ (at a mean of $1350 \mathrm{kIU} / \mathrm{l})$.

\section{Competitive ELISA studies}

Tg-MAbs were produced and characterised at the INSERM U555, Faculté de Médecine, Marseille, France (11). These Tg-MAbs (MAb1-3, 5-11) recognise six antigenic clusters on Tg (I-VI) of which clusters I, III and IV define the immunodominant region typically recognised by AITD sera (15). TgAb epitope recognition was determined in competitive ELISA reactions between alkaline-phosphatase-labelled $\mathrm{Tg}-\mathrm{MAbs}$ and $\mathrm{TgAb}$ in test serum as previously described (15). Briefly, 96-well microtitre plates were coated overnight with $100 \mu \mathrm{l}$ of a $10 \mu \mathrm{g} / \mathrm{ml}$ solution of $\mathrm{Tg}$ in carbonate-bicarbonate buffer. The plates were then washed with PBS-Tween after which $100 \mu \mathrm{l}$ test serum at various dilutions $(1 / 10,1 / 100,1 / 1000)$ was added to the wells and incubated for $2 \mathrm{~h}$ in a humid box at $37^{\circ} \mathrm{C}$. Plates were washed again prior to incubation with $100 \mu$ l labelled $\mathrm{Tg}-\mathrm{MAb}$ for $2 \mathrm{~h}$ in a humid box at $37^{\circ} \mathrm{C}$. After further washing, 4-nitrophenyl phosphate was added to the wells as substrate. Percentage inhibition of MAb binding to $\mathrm{Tg}$ by serum samples was calculated based on the optical densities (OD) at $405 \mathrm{~nm}$ according to the formula:

OD in the absence of serum - OD in the presence of serum OD in the absence of serum

$\times 100$

Inhibition curves for various MAbs were obtained using dilutions of pooled TgAb-positive AITD sera. More than $70 \%$ inhibition was taken as complete inhibition and $35-70 \%$ was interpreted as partial inhibition. OD obtained at 1:100 dilutions was used for analysis.

\section{Statistical analysis}

Data is presented as means (s.D.) except where otherwise indicated. All statistical analysis was performed using SPSS for Windows, version 16.0 (SPSS Inc., Chicago, IL, USA). Continuous data in various groups were compared using the Student's t-test or the MannWhitney $U$ test as appropriate. Recognition of various $\mathrm{Tg}$ clusters was compared between subject groups using the $\chi^{2}$ test with the Bonferroni correction applied for multiple-group comparisons. The level of statistical significance at which the null hypothesis was rejected was chosen as 0.05 . 


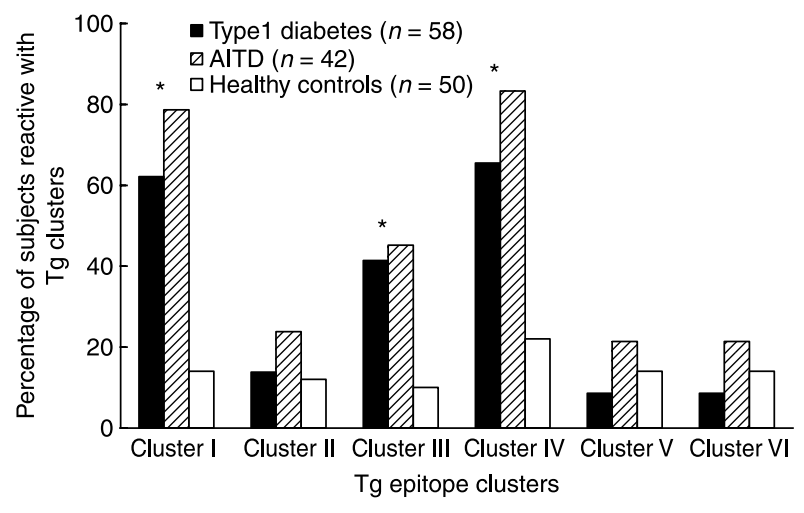

Figure 1 Reactivity with Tg epitope clusters in T1DM, AITD and healthy control subjects. There was a significantly higher preference for immunodominant clusters I, III, IV among subjects with T1DM and AITD compared to healthy controls. Antigenic clusters are labelled I-VI. Cluster I is defined by MAb 6 (J7C73.7), MAb 8 (J8A53.9), MAb 10 (J8A32.13), and MAb 11 (J7B49.15); cluster II is defined by MAb 2 (J8B6.12) and MAb 9 (J8B45.5); cluster III is defined by MAb 3 (J8B89.5) and MAb 5 (J7C44.6); cluster IV is defined by MAb 6 (J7C73.7), MAb 8 (J8A53.9), MAb 10 (J8A32.13), MAb 11 (J7B49.15) and MAb 5 (J7C44.6); cluster V is defined by MAb 1 (J7C9.3); cluster VI is defined by MAb 7 (J7C76.20). Original Tg-MAb nomenclature as characterised by Ruf et al. (11) is in parenthesis. *Healthy controls versus other subject groups.

\section{Results}

\section{Epitope reactivity patterns}

Figure 1 shows the percentage of subjects in each group recognising individual $\mathrm{Tg}$ clusters (I-VI). A subject was said to recognise a $\mathrm{Tg}$ cluster if there was partial (35-70\%) or complete $(>70 \%)$ inhibition of one or more Tg-MAbs within the cluster. There is preferential recognition of the immunodominant clusters I, III and IV by the majority of patients with AITD. On the other hand, only a small percentage of healthy control subjects reacted with these clusters. Among patients with T1DM, the proportion of patients reacting with immunodominant $\mathrm{Tg}$ clusters was not different from AITD patients but was significantly different from that of healthy individuals. The median $\operatorname{TgAb}$ activity in patients with T1DM was significantly lower than in the AITD group (720 vs 1400 IU/l; $P<0.05$ ) but was not different from the healthy control groups (720 vs $620 \mathrm{IU} / \mathrm{l} ; \mathrm{P}>0.05)$.

\section{Clinical characteristics of patients according to epitope reactivity patterns}

Table 1 shows the clinical characteristics of patients according to the epitope reactivity patterns. Patients who showed preferential recognition of one or more Tg-MAbs within the immunodominant clusters were defined as restricted reactivity $(n=44)$, while patients who did not specifically recognise immunodominant clusters were defined as broad reactivity $(n=14)$. There were no differences in age, gender or duration of diabetes in the two groups of patients. Patients with restricted epitope specificities had a higher median TSH and a greater proportion of TPOAb positivity than patients with broad specificities. There was a higher proportion of patients with TSH $>3.0 \mathrm{mU} / \mathrm{l}$ in patients with restricted epitope specificities, although this difference was not statistically significant.

Of the $42 \mathrm{TgAb}$-positive patients with HT, 38 (90.5\%) showed a restricted epitope recognition pattern, while 4 $(9.5 \%)$ showed broad epitope reactivity. All HT patients were TPOAb positive, and median TPOAb concentrations were not significantly different in patients with restricted and broad $\mathrm{Tg}$ reactivity $(680$ vs $770 \mathrm{kIU} / \mathrm{l} ; P>0.05)$.

\section{Discussion}

In this study, we have demonstrated that the majority of TgAb-positive Sri Lankan patients with T1DM preferentially recognise immunodominant $\mathrm{Tg}$ clusters in the same manner as AITD patients. The link between T1DM and thyroid autoimmunity is well established. Both conditions may coexist with or without other autoimmune disorders, and may share common susceptibility genes (16). The similarity in epitope specificities between AITD and T1DM demonstrated in

Table 1 Clinical characteristics of thyroglobulin antibody ( $\operatorname{TgAb})$-positive subjects with type 1 diabetes according to epitope reactivity.

\begin{tabular}{llll}
\hline & Restricted epitope reactivity & Broad epitope reactivity & P value \\
\hline Number of patients & 44 & 14 & - \\
Age at study (years; median (range)) & $16(9-32)$ & $16(5-35)$ & NS \\
Gender (M/F) & $19 / 25$ & $5 / 9$ & $\mathrm{NS}$ \\
Diabetes duration (months; median (range)) & $9.17(0.4-57.1)$ & $11.25(0.4-47.3)$ & $\mathrm{NS}$ \\
$\mathrm{FT}$ (pmol/l) & $17.6 \pm 2.4$ & $17.7 \pm 2.7$ & $\mathrm{NS}$ \\
$\mathrm{TSH}(\mathrm{mU} / \mathrm{l} ;$ median (range)) & $1.60(0.12-3.67)$ & $1.06(0.65-2.04)$ & 0.01 \\
TSH $>3.0 \mathrm{mU} / \mathrm{l}(n(\%))$ & $11(25 \%)$ & $1(7.1 \%)$ & $\mathrm{NS}$ \\
TSH $>5.2 \mathrm{mU} / \mathrm{l}(n(\%))$ & $0(0 \%)$ & $0(0 \%)$ & $\mathrm{NS}$ \\
TPOAb positive $(n(\%))$ & $23(52.3 \%)$ & $1(7.1 \%)$ & 0.004 \\
TgAb level (kIU/l; median (range)) & $810(30-4770)$ & $670(40-3690)$ & $\mathrm{NS}$ \\
\hline
\end{tabular}

A higher median TSH and prevalence of TPOAb was seen in subjects with 'restricted reactivity' (preferential recognition of one or more Tg-MAbs within the immunodominant clusters) compared to subjects with 'broad reactivity' (did not preferentially recognise immunodominant clusters). There was no difference in age, gender or duration of diabetes in the two groups of patients. 
this study may suggest identical immune response pathways to exposed immunodominant epitopes. However, it is likely that expression of epitope-specific $\mathrm{TgAbs}$ signifies co-existent autoimmune thyroiditis in these patients with T1DM.

T1DM patients with narrow epitope specificities had higher TSH levels and were more frequently positive for TPOAbs than those with broad specificities. Longitudinal studies have shown that positive TPOAb status at the onset of diabetes is a strong determinant of future thyroid dysfunction (1). Also, a TSH level in the upper range of normal has been reported to predict thyroid dysfunction in diabetic patients (17). Thus, it is likely that our panel of Tg-MAbs identified those TgAbpositive diabetic patients at risk of thyroid dysfunction. From the clinical perspective, patients with epitoperestricted $\mathrm{TgAbs}$ will benefit from close monitoring and prompt correction of thyroid dysfunction. It is recognised that undiagnosed thyroid dysfunction in diabetic patients could impair glycaemic control and increase cardiovascular risk through adverse effects on body weight and lipid metabolism (18).

Our findings here add to our previous observations in this population. Following iodisation in Sri Lanka, we detected an unusually high prevalence of TgAbs in healthy individuals (6). Most of these TgAbs exhibited broad epitope specificities and disappeared over time, suggesting a non-pathological antibody response to iodisation. On the other hand, TgAb-positive individuals with narrow epitope specifities had persistent autoimmune markers, confirming epitope restriction as a true reflection of thyroid autoimmunity (19). The predominance of epitope-restricted TgAbs in T1DM in this study further supports this distinction between disease-associated and non-specific antibodies.

We acknowledge that our findings in this Sri Lankan population may not necessarily apply to patients with T1DM elsewhere. Studies in stable iodine-replete populations will be instructive in this regard. Also, the relationship between epitope specificities and thyroid function will need to be clarified in longitudinal studies since our study sample included mostly young, newly diagnosed patients who were euthyroid at the time of study. Finally, the overall clinical utility of $\mathrm{TgAb}$ epitope reactivity studies in diagnosing AITD remains limited by its lack of applicability to $\mathrm{TgAb}$-negative patients, some of whom may have AITD. Thus, TPOAb will no doubt remain the important prognostic indicator of AITD. But as we show in this study, Tg epitope specificities may serve a particular role in distinguishing disease-associated antibodies from non-specific antibodies in populations with a high background prevalence of TgAbs.

We conclude that the TgAb epitope specificities in Sri Lankans with T1DM are similar to the pattern seen in autoimmune thyroiditis. These $\mathrm{TgAbs}$ are likely to represent disease-associated antibodies and may potentially identify diabetic patients at risk of thyroid dysfunction.

\section{Declaration of interest}

All authors confirm that there are no conflicts of interest and that there are no financial disclosures to make.

\section{Funding}

This research did not receive any specific grant from any funding agency in the public, commercial or not-for-profit sector.

\section{Acknowledgements}

We thank Dr Jean Ruf for the kind gift of thyroglobulin monoclonal antibodies. We are indebted to Mrs Lynn Taylor for her laboratory assistance.

\section{References}

1 Umpierrez GE, Latif KA, Murphy MB, Lambeth HC, Stentz F, Bush A \& Kitabchi AE. Thyroid dysfunction in patients with type 1 diabetes: a longitudinal study. Diabetes Care 200326 1181-1185.

2 Weetman AP \& McGregor AM. Autoimmune thyroid disease: further developments in our understanding. Endocrine Reviews $199415788-830$.

3 Burek CL, Rose NR, Guire KE \& Hoffman WH. Thyroid autoantibodies in black and in white children and adolescents with type 1 diabetes mellitus and their first degree relatives. Autoimmunity 19907 157-167.

4 Hollowell JG, Staehling NW, Flanders WD, Hannon WH, Gunter EW, Spencer CA \& Braverman LE. Serum TSH, T 4 , and thyroid antibodies in the United States population (1988 to 1994): National Health and Nutrition Examination Survey (NHANES III). Journal of Clinical Endocrinology and Metabolism 2002 87 489-499.

5 Marwaha RK, Tandon N, Karak AK, Gupta N, Verma K \& Kochupillai N. Hashimoto's thyroiditis: countrywide screening of goitrous healthy young girls in postiodization phase in India. Journal of Clinical Endocrinology and Metabolism $2000 \mathbf{8 5}$ 3798-3802.

6 Premawardhana LD, Parkes AB, Smyth PP, Wijeyaratne CN, Jayasinghe A, De Silva DGH \& Lazarus JH. Increased prevalence of thyroglobulin antibodies in Sri Lankan schoolgirls - is iodine the cause? European Journal of Endocrinology 2000143 185-188.

7 Premawardhana LD, Wijeyaratne $\mathrm{CN}$, Chen S, Wijesuriya M, Illangasekara U, Brooking H, Amoroso M, Jeffreys J, Bolton J, Lazarus JH, Furmaniak J \& Rees Smith B. Thyroid, adrenal and celiac disease related autoantibodies in patients with type 1 diabetes from Sri Lanka. Journal of Endocrinological Investigation 200629 968-974.

8 Caturegli P, Mariotti S, Kuppers RC, Burek CL, Pinchera A \& Rose NR. Epitopes on thyroglobulin: a study of patients with thyroid disease. Autoimmunity 199418 41-49.

9 Saboori AM, Rose NR, Yuhasz SC, Amzel LM \& Burek CL. Peptides of human thyroglobulin reactive with sera of patients with autoimmune thyroid disease. Journal of Immunology 1999163 6244-6250.

10 Dunn JT. Thyroglobulin: chemistry and biosynthesis. In Werner and Ingbar's the Thyroid, ch 5, pp 85-95. Eds LE Braverman \& RD Utiger. Philadelphia: JB Lippincott Company, 1996.

11 Ruf J, Carayon P, Sarles-Philip S, Kourilsky F \& Lissitzky S. Specificity of monoclonal antibodies against human thyroglobulin; comparison with autoimmune antibodies. EMBO Journal 19832 $1821-1826$.

12 Bresler HS, Burek CL, Hoffman W \& Rose NR. Autoantigenic determinants on human thyroglobulin. II. Determinants recognized by autoantibodies from patients with chronic autoimmune thyroiditis compared to autoantibodies from healthy subjects. Clinical Immunology and Immunopathology $1990 \mathbf{5 4}$ 76-86. 
13 Alberti KG \& Zimmet PZ. Definition, diagnosis and classification of diabetes mellitus and its complications. Part 1: diagnosis and classification of diabetes mellitus provisional report of a WHO consultation. Diabetic Medicine 199815 539-553.

14 Groves CJ, Howells RD, Williams S, Darke C \& Parkes AB. Primary standardization for the ELISA of serum thyroperoxidase and thyroglobulin antibodies and their prevalence in a normal Welsh population. Journal of Clinical \& Laboratory Immunology 199032 147-151.

15 Okosieme OE, Premawardhana LD, Jayasinghe A, De Silva DGH, Smyth PP, Parkes AB, Lejeune PJ, Ruf J \& Lazarus JH. Thyroglobulin epitope recognition in a post iodine-supplemented Sri Lankan population. Clinical Endocrinology 200359 190-197.

16 Barker JM. Type 1 diabetes-associated autoimmunity: natural history, genetic associations, and screening. Journal of Clinical Endocrinology and Metabolism 200691 1210-1217.

17 Warren RE, Perros P, Nyirenda MJ \& Frier BM. Serum thyrotropin is a better predictor of future thyroid dysfunction than thyroid autoantibody status in biochemically euthyroid patients with diabetes: implications for screening. Thyroid 2004 14 853-857.

18 Mohn A, Di Michele S, Di Luzio R, Tumini S \& Chiarelli F. The effect of subclinical hypothyroidism on metabolic control in children and adolescents with type 1 diabetes mellitus. Diabetic Medicine 2002 $1970-73$.

19 Okosieme OE, Premawardhana LD, Jayasinghe A, Kaluarachi WN, Parkes AB, Smyth PP, Lejeune PJ, Ruf J \& Lazarus JH. Thyroglobulin autoantibodies in iodized subjects: relationship between epitope specificities and longitudinal antibody activity. Thyroid 200515 1067-1072.

Received 18 June 2009

Accepted 24 June 2009 